\title{
A new approach for testing variations of fundamental constants over cosmic epochs using FIR fine-structure lines
}

\author{
S. A. Levshakov ${ }^{1, \star}$, D. Reimers ${ }^{1}$, M. G. Kozlov ${ }^{2,1}$, S. G. Porsev ${ }^{2,1}$, and P. Molaro ${ }^{3}$ \\ 1 Hamburger Sternwarte, Universität Hamburg, Gojenbergsweg 112, 21029 Hamburg, Germany \\ e-mail: lev@astro.ioffe.rssi.ru \\ 2 Petersburg Nuclear Physics Institute, Gatchina, 188300, Russia \\ 3 Osservatorio Astronomico di Trieste, via G. B. Tiepolo 11, 34131 Trieste, Italy
}

Received 21 November 2007 / Accepted 7 December 2007

\begin{abstract}
Aims. We aim to obtain limits on the variation of the fine-structure constant $\alpha$ and the electron-to-proton mass ratio $\mu$ over different cosmological epochs.

Methods. A new approach based on the comparison of redshifts of far infrared (FIR) fine-structure lines and low-lying rotational transitions in $\mathrm{CO}$ is proposed which is in principle more sensitive by a factor of $\sim 10$ than QSO metal absorption lines.

Results. Estimations of the quotient $F=\alpha^{2} / \mu$ obtained for two distant quasars J1148+5251 $(z=6.42)$ and BR 1202-0725 $(z=4.69)$ provide $\Delta F / F=(0.1 \pm 1.0) \times 10^{-4}$ and $(1.4 \pm 1.5) \times 10^{-4}$. The obtained limits are consistent with no variation of physical constants at the level of $\sim 0.01 \%$ over a period of $13 \mathrm{Gyr}$.

Conclusions. Upcoming observations of quasars and distant galaxies in FIR fine-structure lines of different species and in CO low rotational lines at the SOFIA, HSO, and ALMA are expected to improve the current limit by, at least, an order of magnitude.
\end{abstract}

Key words. cosmology: observations - line: profiles - galaxies: quasars: absorption lines

\section{Introduction}

Possible time variation of coupling strengths and particle masses is now being discussed with regard to the accelerating expansion of the Universe. Theoretical models imposing extra dimensions predict that dimensionless quantities like the fine-structure constant, $\alpha=\mathrm{e}^{2} /(\hbar c) \approx 1 / 137$, the electron-to-proton mass ratio, $\mu=m_{\mathrm{e}} / m_{\mathrm{p}}$, etc. depend on the scale length of extra dimensions in Kaluza-Klein or superstring theories (for a review, see García-Berro et al. 2007). This scale factor may vary with cosmic time giving rise to variations of fundamental constants which are defined in the combined 4D and extra-D space-time. Very different patterns, from linear and slow-rolling to oscillating variations, are considered in contemporary theoretical models (e.g., Marciano 1984; Mota \& Barrow 2004; Fujii 2005). But until now, the evidence for any changes in fundamental constants has not been unambiguously asserted.

For example, laboratory measurements do not show variations of $\alpha$ at the level of $\dot{\alpha} / \alpha=(-2.6 \pm 3.9) \times 10^{-16} \mathrm{yr}^{-1}$ (Peik et al. 2006). A lower limit can be set by the fission product analysis of a natural reactor in Oklo (in units of $10^{-17} \mathrm{yr}^{-1}$ ): $-0.6 \leq \dot{\alpha} / \alpha \leq 1.2$ and $-4 \leq \dot{\alpha} / \alpha \leq 3$ reported by Gould et al. (2006) and Petrov et al. (2006) which approximately corresponds to the redshift $z \simeq 0.14$. At higher redshifts, molecular rotational and atomic resonance transitions in combination with observations of the HI $21 \mathrm{~cm}$ hyperfine line were used to constrain different combinations of physical constants (e.g., Varshalovich \& Potekhin 1996; Drinkwater et al. 1998; Carilli et al. 2000; Murphy et al. 2001; Kanekar et al. 2005; Tzanavaris et al. 2007). A new constraint on $\Delta \mu / \mu$ at $z=0.68$,

* On leave from the Ioffe Physico-Technical Institute, St. Petersburg, Russia.
$\Delta \mu / \mu=-0.6 \pm 1.9 \mathrm{ppm}(1 \sigma$ statistical error $)$ was recently reported by Flambaum \& Kozlov (2007) from the comparison of absorption-line spectra of $\mathrm{NH}_{3}$ with $\mathrm{CO}, \mathrm{HCO}^{+}$, and $\mathrm{HCN}$ in the radio frequency region ${ }^{1}$. This value of $\Delta \mu / \mu$ being linear extrapolated to $z=0$ corresponds to $\dot{\mu} / \mu=(-1 \pm 3) \times 10^{-16} \mathrm{yr}^{-1}$. Here we adopt a cosmology with $H_{0}=70 \mathrm{~km} \mathrm{~s}^{-1} \mathrm{Mpc}^{-1}, \Omega_{\Lambda}=0.7$, and $\Omega_{\mathrm{m}}=0.3$ (look-back time $t_{z}=6.3 \mathrm{Gyr}$ at $z=0.68$ ).

In optical spectra of QSOs, limits on $\Delta \alpha / \alpha$ of comparable accuracy can be obtained from metal absorption line measurements. Special observations aimed at such measurements were performed with two different spectrographs - the HARPS at the ESO 3.6-m telescope (Chand et al. 2006) and the UVES at the ESO 8-m telescope (Levshakov et al. 2007a). The following values for $\Delta \alpha / \alpha$ were obtained (Levshakov et al. 2007b): $-0.12 \pm 1.79 \mathrm{ppm}$ at $z=1.15$ and $5.66 \pm 2.67 \mathrm{ppm}$ at $z=1.84$ $(1 \sigma$ errors including statistical and systematic parts are indicated). Assuming linear variation with cosmic time, one finds $\dot{\alpha} / \alpha=(-0.1 \pm 2.1) \times 10^{-16} \mathrm{yr}^{-1}$ and $\dot{\alpha} / \alpha=(5.5 \pm 2.6) \times 10^{-16} \mathrm{yr}^{-1}$, respectively.

To test possible systematics in $\Delta \alpha / \alpha$ measurements with the UVES spectrograph, we performed additional control observations of asteroids (Molaro et al. 2007). Asteroids provide an accurate radial velocity reference at the level of $1 \mathrm{~m} \mathrm{~s}^{-1}$, or $0.03 \mathrm{ppm}$ in units of $\Delta \alpha / \alpha$. Since no systematic shifts larger than $1.5 \mathrm{ppm}$ have been revealed, the positive signal at $z=1.84$ may be real or be induced by other yet unknown systematics.

At higher redshifts, $z \gtrsim 3.5$, the changes in fundamental constants can be probed through observations of distant galaxies and QSOs in the far infrared (FIR) and radio ranges. Carbon,

\footnotetext{
1 Note that $\mu=m_{\mathrm{p}} / m_{\mathrm{e}}$ in Flambaum \& Kozlov (2007).

$1 \mathrm{ppm} \equiv 10^{-6}$.
} 
nitrogen and oxygen FIR fine-structure lines were suggested to probe the interstellar medium in galaxies at cosmological distances and to search for $z>10$ objects (Petrosian et al. 1969; Loeb 1993; Stark 1997; Suginohara et al. 1999; Blain et al. 2000; Boselli et al. 2002; Papadopoulos et al. 2004; Schaeres \& Pelló 2005; Nagamine et al. 2006).

In this paper we report on the limits on $\alpha^{2} / \mu$ estimated for the first time from the [C II] $158 \mu \mathrm{m}$ line combined with $\mathrm{CO}$ rotational lines detected in the spectra of the most distant quasar $\mathrm{J} 1148+5251$ at a redshift $z=6.42$ (Maiolino et al. 2005) and in the northern component of a pair of AGN hosts BR 1202-0725 at $z=4.69$ (Iono et al. 2006). The corresponding look-back time is $t_{z}=12.9 \mathrm{Gyr}$ and $12.5 \mathrm{Gyr}$ which is, respectively, $93 \%$ and $91 \%$ of the age of the Universe.

\section{Computational method}

In the nonrelativistic limit and for an infinitely heavy point-like nucleus all atomic transition frequencies are proportional to the Rydberg constant, $\mathcal{R} \approx 109737.3 \mathrm{~cm}^{-1}$. In this approximation, the ratio of any two atomic frequencies does not depend on any fundamental constant. Relativistic effects lead to the corrections to an atomic energy which are proportional to the product $(\alpha Z)^{2} \mathcal{R}$, where $Z$ is atomic number. Corrections accounting for the finite nuclear mass are proportional to $\mu \mathcal{R}$. For atoms these corrections are typically much smaller than relativistic corrections, but they become important for molecules.

To study the dependence of atomic frequencies on $\alpha$ it is convenient to expand them near the present-time value of $\alpha$ in the co-moving reference frame:

$\omega_{\mathrm{z}}=\omega+q x+\ldots, \quad x \equiv\left(\alpha_{\mathrm{z}} / \alpha\right)^{2}-1$.

Here $\omega$ and $\omega_{z}$ are the frequencies corresponding to the present value of $\alpha$ and to a change $\alpha \rightarrow \alpha_{z}$ presumably at epoch $z$. The parameter $q$ (so-called $q$-factor) is individual for each atomic transition (Dzuba et al. 2002).

If $\alpha$ is not a constant, the parameter $x$ differs from zero. In a linear approximation, $|\Delta \alpha / \alpha| \ll 1$, the corresponding frequency shift, $\Delta \omega=\omega_{z}-\omega$, is given by:

$\Delta \omega / \omega=2 Q \Delta \alpha / \alpha$,

where $Q=q / \omega$ is the dimensionless sensitivity coefficient, and $\Delta \alpha=\alpha_{z}-\alpha$.

For distant objects such a frequency shift would cause an apparent change in the redshift

$\Delta \omega / \omega=-\Delta z /(1+z)$,

where $\Delta z=\tilde{z}-z$ is the difference between an apparent redshift $\tilde{z}$ and its true value $z$. If $\omega^{\prime}$ is the observed frequency from a distant object, then the true redshift is given by

$1+z=\omega_{z} / \omega^{\prime}$

whereas the shifted (apparent) value is

$1+\tilde{z}=\omega / \omega^{\prime}$.

Relativistic corrections grow with atomic number $Z$, but for optical and UV transitions in light elements they are small: $Q \sim(\alpha Z)^{2} \ll 1$. For example, the UV Fe II lines used in the $\Delta \alpha / \alpha$ measurements have sensitivities $|Q| \sim 0.03$ (Porsev et al. 2007). All other metal lines usually observed in quasar spectra have even lower values of $Q$ except for some of the Fe I or Zn II transitions (Dzuba et al. 2002; Dzuba \& Flambaum 2007), which are, however, too weak to be suitable for precise measurements of redshifts from quasar spectra.

However, in the radio and FIR ranges one can significantly increase the sensitivity to $\alpha$-variation by looking at transitions between the fine-structure levels. In the nonrelativistic limit $(\alpha \rightarrow 0)$ such levels are exactly degenerated. The corresponding transition frequencies $\omega_{\mathrm{fs}}$ are proportional to $(\alpha Z)^{2}$ which means that for these transitions $Q=1$, i.e. they are about 30 times more sensitive to changes in $\alpha$ than UV lines. This gives

$\Delta \omega_{\mathrm{fs}} / \omega_{\mathrm{fs}}=2 \Delta \alpha / \alpha$

In order to utilize the high sensitivity of the fine-structure transitions in differential measurements of $\alpha$, we need an independent reference source. Good candidates for this purpose are lowlying rotational lines of $\mathrm{CO}$ : carbon monoxide is the second most abundant molecule after $\mathrm{H}_{2}$; it is observed in many extragalactic objects ${ }^{2}$, and frequencies of the rotational transitions of $\mathrm{CO}$ (hundreds $\mathrm{GHz}$ ) fall close to the frequency of the [C II] finestructure transition ${ }^{2} \mathrm{P}_{3 / 2} \rightarrow{ }^{2} \mathrm{P}_{1 / 2}$ at $1900.539 \mathrm{GHz}$.

To a good approximation, the frequencies $\omega_{\text {rot }}$ of the rotational lines of light molecules are independent of $\alpha$, but sensitive to the electron to proton mass ratio $\mu$ : $\omega_{\text {rot }} \sim \mu \mathcal{R}$, i.e.

$\Delta \omega_{\text {rot }} / \omega_{\text {rot }}=\Delta \mu / \mu$.

If $\alpha$ and/or $\mu$ changes over cosmic time, the apparent redshifts for the fine-structure line(s) and for the rotational line(s) will differ. Using Eqs. (3), (6), and (7), one finds

$\left(\tilde{z}_{\mathrm{rot}}-\tilde{z}_{\mathrm{fs}}\right) /(1+z) \equiv \Delta z /(1+z)=2 \Delta \alpha / \alpha-\Delta \mu / \mu$,

where $z$ is a reference redshift.

By introducing the parameter $F=\alpha^{2} / \mu$, we can rewrite Eq. (8) in the form

$\Delta z /(1+z)=\Delta F / F$.

Variation of $\mu$ is by far larger than that of $\alpha$, as predicted by some GUT models (e.g. Langacker et al. 2002; Flambaum 2007).

\section{Results}

Now we apply Eq. (9) to the observations of [C II] $158 \mu \mathrm{m}$ and $\mathrm{CO}$ emission lines from the quasars $\mathrm{J} 1148+5251$ and BR 1202-0725 to obtain a limit on variation of the parameter $F$.

The spectral observations of the [C II] $158 \mu \mathrm{m}$ emission line in the quasar J1148+5251 were carried out with the Institut de Radioastronomie Millimétrique (IRAM) 30-m telescope at the frequency of $256.1753 \mathrm{GHz}$ covering a bandwidth of $1 \mathrm{GHz}$ with 256 channels spaced by $4 \mathrm{MHz}$ (Maiolino et al. 2005). At this frequency the resolving power of the telescope is 9.6 arcsec (Half Power Beam Width) and the $1 \mathrm{GHz}$ bandwidth corresponds to $1170 \mathrm{~km} \mathrm{~s}^{-1}$. The resulting spectral resolution and the noise in the coadded and rebinned spectrum were, respectively, $56 \mathrm{~km} \mathrm{~s}^{-1}$ and $0.3 \mathrm{mK}(2.8 \mathrm{mJy})$, leading to a $30 \%$ accuracy of the flux density scale. The [C II] line was detected at a significance level of $8 \sigma$ for the total exposure time of $12.4 \mathrm{~h}$. The redshift and the peak intensity of the [C II] line are $z_{\mathrm{fs}}=6.4189 \pm 0.0006$ and $I_{158}=11.8 \mathrm{mJy}$. The reported error $\sigma_{z}=0.0006$ corresponds to the uncertainty of the line position

2 Emission lines from the rotational transitions of $\mathrm{CO}$ were detected in $\sim 40$ galaxies at $z>1$ (for a review, see Solomon \& Vanden Bout 2005), and recently at $z=5.0267$ towards SDSS J033829.31+002156.3 (Maiolino et al. 2007) and $z=5.7722$ towards SDSS J0927+2001 (Carilli et al. 2007). 
measurement of $\sigma_{\mathrm{v}} \sim 24 \mathrm{~km} \mathrm{~s}^{-1}$, which is about one bin size in the $[\mathrm{C}$ II] spectrum at the Nyquist limit of 2 resolution elements.

Observations of the $\mathrm{CO}(7 \rightarrow 6)$ and $(6 \rightarrow 5)$ emission lines were obtained with the IRAM Plateau de Bure interferometer at the frequencies $108.724 \mathrm{GHz}$ (the total integration time $\left.T_{\exp }=22 \mathrm{~h}\right)$ and $93.206 \mathrm{GHz}\left(T_{\exp }=14 \mathrm{~h}\right)$, respectively (Bertoldi et al. 2003). At about 5 arcsec angular resolution $\left(5.7^{\prime \prime} \times 4.1^{\prime \prime}\right.$ at $\left.3.2 \mathrm{~mm}\right)$ the $\mathrm{CO}$ emission line is unresolved and coincides within the astrometric uncertainties of $\pm 0.3 \operatorname{arcsec}$ with the optical position of the quasar given by Fan et al. (2003). The coadded $3 \mathrm{~mm}$ data were rebinned to $64 \mathrm{~km} \mathrm{~s}^{-1}(J=7 \rightarrow 6)$ and $55 \mathrm{~km} \mathrm{~s}^{-1}(J=6 \rightarrow 5)$ resulting in an accuracy of the line position measurements of $\sigma_{\mathrm{v}} \sim 36$ and $24 \mathrm{~km} \mathrm{~s}^{-1}$, respectively. These uncertainties are again of a bin size in the reduced spectra. The redshifts and the peak intensities of the $\mathrm{CO}(7 \rightarrow 6)$ and $(6 \rightarrow 5)$ lines are, respectively, $z_{\mathrm{rot}}^{(7-6)}=6.4192 \pm 0.0009, I_{(7-6)}=$ $2.14 \mathrm{mJy}$, and $z_{\text {rot }}^{(6-5)}=6.4189 \pm 0.0006, I_{(6-5)}=2.45 \mathrm{mJy}$.

Weighting the reported rotational redshifts by these peak intensities, one obtains the mean $z_{\text {rot }}=6.4190 \pm 0.0005$. We will take this value for the quasar's systemic redshift $z$. Using the reported redshift $z_{\mathrm{fs}}$ and the averaged $z_{\text {rot }}$, Eq. (9) yields $\Delta F / F=(0.1 \pm 1.0) \times 10^{-4}$.

The second [C II] line was detected at $z_{\mathrm{fs}}=4.6908$ towards the northern component of the quasar BR 1202-0725 (Iono et al. 2006). The profile of this line is similar to the $\mathrm{CO}(5 \rightarrow 4)$ and $(7 \rightarrow 6)$ lines seen at $z_{\text {rot }}=4.6916$ from the same component (Omont et al. 1999).

The $[\mathrm{CII}] 158 \mu \mathrm{m}$ emission was observed with the Submillimeter Array interferometer (SMA, Ho et al. 2004). The total exposure time at a redshifted [C II] frequency of $333.969 \mathrm{GHz}$ was $T_{\exp }=19.6 \mathrm{~h}$, and the angular resolution was $3.4^{\prime \prime} \times 2.7^{\prime \prime}$. The coadded spectrum was averaged using a $120 \mathrm{~km} \mathrm{~s}^{-1}$ bin size resulting in the rms noise of $7.5 \mathrm{mJy}$, or the signal-to-noise ratio $S / N \sim 3$ (the peak flux density $\sim 23 \mathrm{mJy}$ as shown in Fig. 1 in Iono et al.). Assuming the uncertainty of the $[\mathrm{CII}]$ line position as $\sim 1 / 4$ bin size, one gets the error $\sigma_{\mathrm{v}} \simeq 30 \mathrm{~km} \mathrm{~s}^{-1}$.

The CO $J=5 \rightarrow 4$ line observed with the IRAM interferometer is detected at the $\sim 5 \sigma$ confidence level $\left(T_{\exp } \sim 16 \mathrm{~h}\right)$, and the CO $J=7 \rightarrow 6$ line observed with the IRAM 30-m telescope at the $3 \sigma$ confidence level. The angular resolution in the interferometric observations was $5.0 \times 2.5$ arcsec, and velocity resolution of about $60 \mathrm{~km} \mathrm{~s}^{-1}$. The redshift of the northern component is $z_{5-4}=4.6916$. The uncertainty of this value is, probably, 25-30 $\mathrm{km} \mathrm{s}^{-1}$, i.e. approximately one resolution element, considering the rather noisy line profiles shown in Fig. 2 in Omont et al. The angular resolution of the 30-m telescope for the $2-\mathrm{mm}$ beam is $17 \mathrm{arcsec}$. The error of the reported redshift $z_{7-6}=4.6915 \pm 0.001$ corresponds to the radial velocity uncertainty of $53 \mathrm{~km} \mathrm{~s}^{-1}$.

Taking into account that the angular resolutions are similar in observations of the $[\mathrm{C} \mathrm{II}]$ and $\mathrm{CO} J=5 \rightarrow 4$ emission lines, we can use their redshifts $z_{\mathrm{fs}}=4.6908 \pm 0.0006$ and $z_{\mathrm{rot}}=4.6916 \pm$ 0.0006 (both errors correspond to $\sigma_{\mathrm{v}}=30 \mathrm{~km} \mathrm{~s}^{-1}$ ) to calculate $\Delta F / F=(1.4 \pm 1.5) \times 10^{-4}$.

\section{Discussion}

While comparing the redshifted frequencies of different species to measure hypothetical variations of physical constants, one must account for random Doppler shifts of the line positions caused by non-identical spatial distributions of species (referred to as the Doppler noise hereafter) which can mimic non-zero signals in $\Delta \alpha / \alpha$ or $\Delta \mu / \mu$ or in a combination of these quantities (e.g., Levshakov 1994; Carilli et al. 2000; Bahcall et al. 2004). To quantify uncertainties induced by the Doppler noise a sample of $(\Delta \alpha / \alpha) /(\Delta \mu / \mu)$ measurements should be collected.

The main problem here is how to estimate the dispersion of random velocity shifts $\sigma_{\mathrm{v}}$ for a given system of spectral lines. In the case of a large sample size the value of $\sigma_{\mathrm{v}}$ can be found from the scatter of points. For a single measurement, a guess for $\sigma_{\mathrm{v}}$ comes from the comparison with data on velocity differences between spectral lines of similar species in nearby clouds. Observations of local galaxies show that the intensity of [C II] is strongly correlated with the intensities of the low-lying rotational lines of CO, the fine-structure lines of [C I ] $\lambda \lambda 370,609 \mu \mathrm{m}$ and [OI] $\lambda \lambda 63,146 \mu \mathrm{m}$ (Malhotra et al. 2001), and the finestructure line of [N II] $\lambda 205 \mu \mathrm{m}$ (Petuchowski \& Bennett 1993; Abel 2006). However, the surface distribution of the [C II] emission may not precisely follow the actual ${ }^{12} \mathrm{CO}$ contours (Stacey et al. 1985). The CO rotational lines, if optically thin, are emitted throughout the whole molecular cloud. As for the [C II] emission, it is usually enhanced at the edges of the molecular cloud in the photodissociation regions (PDRs). Additionally, diffuse gas from the $\mathrm{H}$ II regions can contribute to the intensity of the "PDR" lines (Kaufman et al. 1999). However, the impact from the diffuse gas decreases with increasing gas densities and drops from $\sim 30 \%$ at $n_{\mathrm{H}} \sim 1 \mathrm{~cm}^{-3}$ to $\sim 10 \%$ at $n_{\mathrm{H}} \sim 10^{3} \mathrm{~cm}^{-3}$ (Kaufman et al. 2006).

Because both considered high-redshift [C II] emitters belong to the giant molecular gas complexes with $M \sim 10^{10} M_{\odot}($ Omont et al. 1999; Walter et al. 2004), the contribution to the integrated observed intensity of the [C II] emission from the H II regions is probably negligible. In particular, Iono et al. (2006) note an excellent agreement between the $[\mathrm{C} \mathrm{II}]$ and $\mathrm{CO}$ profiles at $z=$ 4.69. At $z=6.42$, Maiolino et al. (2005) favor $n_{\mathrm{H}} \sim 10^{5} \mathrm{~cm}^{-3}$ to explain the observed luminosities in FIR, $\mathrm{CO}$, and [C II] lines.

Our estimate of the value of the Doppler noise for the $\mathrm{CO}$ and [C II] emission lines at $z \sim 6$ is based on typical parameters of the interstellar molecular clouds with similar gas densities: $n=10^{2}-10^{4} \mathrm{~cm}^{-3}, M=10^{2}-10^{4} M_{\odot}$, size $L=2-20 \mathrm{pc}$ (Table 1 in Mac Low \& Klessen 2004). Using the velocity dispersion and region size relation, $\sigma_{\mathrm{v}}\left(\mathrm{km} \mathrm{s}^{-1}\right)=1.10 L^{0.38}(\mathrm{pc})$, empirically derived for molecular clouds by Larson (1981) and then studied in detail by Solomon et al. (1987) and by other groups (e.g., Falgarone et al. 1992; Miesch \& Bally 1994; Caselli \& Myers 1995; Frieswijk et al. 2007), we find $\sigma_{\mathrm{v}} \sim 1-3 \mathrm{~km} \mathrm{~s}^{-1}$. Thus, we may assume that if the $[\mathrm{C}$ II $]$ and $\mathrm{CO}$ lines arise cospatially, then the velocity offset between them does not exceed the velocity dispersion within the cloud which is of the order of a few $\mathrm{km} \mathrm{s}^{-1}$. On the other hand, our derived limit $|\Delta F / F|<10^{-4}$ corresponds to $\sigma_{\mathrm{v}}=30 \mathrm{~km} \mathrm{~s}^{-1}$ which is the error of the line position measurement. From these values, we are confident that the quotient $F=\alpha^{2} / \mu$ remains constant at the level of $0.01 \%$ to a look-back time of 13 Gyr.

The reliability of the result obtained can be compared with previous radio observations of molecules at intermediate redshifts. Kanekar et al. (2005) used observations of H I $21 \mathrm{~cm}$ and $\mathrm{OH} 18 \mathrm{~cm}$ lines from two absorbers at $z \simeq 0.7$ and found $\Delta f / f=\left(0.44 \pm 0.36_{\text {stat }} \pm 1.0_{\text {syst }}\right) \times 10^{-5}$. Here $f=g_{\mathrm{p}}\left(\alpha^{2} / \mu\right)^{1.57}$, and $g_{\mathrm{p}}$ is the proton gyromagnetic ratio. The systematic error was set from the assumption that the Doppler noise is $3 \mathrm{~km} \mathrm{~s}^{-1}$. However, this seems to be too optimistic taking into account observations of the nearby diffuse/translucent gas clouds in our Galaxy. For instance, absorption profiles of the $\mathrm{OH} 18 \mathrm{~cm}$ and $\mathrm{HCO}^{+} 3 \mathrm{~mm}$ lines studied at $140-240 \mathrm{~m} \mathrm{~s}^{-1}$ resolution towards extragalactic compact continuum sources (Liszt \& Lucas 2000) 
exhibit, in general, similar complex structures over the radial velocity interval of $\sim 10-20 \mathrm{~km} \mathrm{~s}^{-1}$, while showing small differences in details on the scale of $1-2 \mathrm{~km} \mathrm{~s}^{-1}$. However, comparison of $\mathrm{HCO}^{+}$absorption with $\mathrm{H}$ I $21 \mathrm{~cm}$ absorption towards the same targets reveals significant discrepancies. Figures 6 and 7 from Liszt \& Lucas show that there are rather wide velocity intervals $\left(\sim 20 \mathrm{~km} \mathrm{~s}^{-1}\right)$ where $\mathrm{HI}$ absorption is seen but $\mathrm{HCO}^{+}$ is absent. It seems quite possible that the Doppler noise for a sample of H I $21 \mathrm{~cm}$ and molecular lines could be as large as $10 \mathrm{~km} \mathrm{~s}^{-1}$. Another set of observations of local warm gas clouds within 15 pc around the Sun (Redfield \& Linsky 2008) show that the distribution of cloud velocity difference has a width of $\sim 10 \mathrm{~km} \mathrm{~s}^{-1}$. This means that in lower resolution observations of quasar intervening systems the line-of-sight velocity differences of the order of $10 \mathrm{~km} \mathrm{~s}^{-1}$ may occur on a rather short linear scale of $\sim 10-100 \mathrm{pc}$.

The limit on $\Delta \mu / \mu=-0.6 \pm 1.9$ ppm by Flambaum \& Kozlov (2007) was estimated from the $\mathrm{NH}_{3}$ transitions near $\lambda=1.26 \mathrm{~cm}$ and $\mathrm{mm}$-wave lines of $\mathrm{CO}, \mathrm{HCO}^{+}$, and $\mathrm{HCN}$ detected from one absorption-line system. In this case $\Delta \mu / \mu=0.289 \Delta v / c$, which means that the uncertainty of $2 \mathrm{ppm}$ corresponds to $\Delta v \simeq$ $2 \mathrm{~km} \mathrm{~s}^{-1}$. As discussed above, the systematic error from the Doppler noise can be of the same order or even larger.

To avoid the influence of the Doppler noise on differential measurements of physical constants, lines of only one element should be utilized. For instance, ground state FIR fine-structure transitions of [C I] $\lambda \lambda 370,609 \mu \mathrm{m},[\mathrm{OI}] \lambda \lambda 63,146 \mu \mathrm{m},[\mathrm{N}$ II] $\lambda \lambda 122,205 \mu \mathrm{m}$, [O III] $\lambda \lambda 52,88 \mu \mathrm{m}$, and [Fe II] $\lambda \lambda 15,26 \mu \mathrm{m}$ can be used to directly constrain $\Delta \alpha / \alpha$. The sensitivity of this procedure to $\alpha$-variation will be discussed in a subsequent paper.

Up to now, [C I] was detected in five high- $z$ objects where $\mathrm{CO}$ has been observed: $\mathrm{H} 1413+117, J=1-0$ line (Barvainis et al. 1997; Weiß et al. 2005) and $J=2-1$ line (Weiß et al. 2003) at $z=2.557$; IRAS F10214+4724 and SMM J14011+0252, $J=1-0$ line at $z=2.285$ and 2.565 , respectively (Weiß et al. 2005); APM $08279+5255, J=1-0$ line at $z=3.913$ (Wagg et al. 2006); PSS $2322+1944, J=1-0$ line at $z=4.120$ (Pety et al. 2004). Unfortunately, low signal-to-noise $(\$ 10)$ and large uncertainties of the positions of $[\mathrm{CI}]$ and CO lines $\left(\sim 25 \mathrm{~km} \mathrm{~s}^{-1}\right)$ prevent us from constraining relative changes in constants with an accuracy better than $10^{-4}$.

\section{Conclusions}

In this letter we propose to use the FIR fine-structure lines of atoms and ions in conjunction with low-lying rotational lines of $\mathrm{CO}$ to probe the cosmic time evolution of the fundamental physical constants, namely the quotient $F=\alpha^{2} / \mu$.

The reported constraints on $\Delta F / F$ are obtained for two [C II] emitters recently discovered at $z=6.42$ (Maiolino et al. 2005) and $z=4.69$ (Iono et al. 2006). We found no evidence for the variability of $F$ at the level of $10^{-4}$ over a period of $13 \mathrm{Gyr}$. The statistical reliability of this limit seems to be high enough since the expected value of the Doppler noise for the co-spatially distributed [C II] and CO emission lines, $\sigma_{\mathrm{v}} \sim$ a few $\mathrm{km} \mathrm{s}^{-1}$, is less that the error of the line position measurement, $\sigma_{\mathrm{v}}=30 \mathrm{~km} \mathrm{~s}^{-1}$. However, to be completely confident it should be tested whether the empirical relationship between the velocity dispersion and region size derived on base of local measurements is also applicable to high- $z$ galaxies.

The result obtained is to be compared with other tests at extremely high redshifts: bounds from the height and the positions of the first and subsequent acoustic peaks of the spectrum of the cosmic microwave background radiation fluctuations $(z \sim 1500$, look-back time $13.8 \mathrm{Gyr}$ ), and from the relative abundances of light elements predicted in the big bang nucleosynthesis $\left(z \sim 10^{10}\right)$ restrict the fine-structure constant variations at the level of only 2-3\% (Ichikawa et al. 2006; Dent et al. 2007; Mosquera et al. 2008). Thus, the proposed method ensures a significant gain in accuracy and works at time scales comparable with the total age of the Universe.

The limits on $\Delta F / F$ can be improved by measuring the line positions with better precision - as high as $1-3 \mathrm{~km} \mathrm{~s}^{-1}$ to be comparable with the expected Doppler noise. The advantage of radio observations for such precise spectral measurements is twofold: firstly, distant objects inaccessible to optical observations can be probed, and, secondly, spectral resolution available in the cmand mm-range is much higher than in the optical. Additionally, the sensitivity to $\alpha$-variation in the FIR band is further increased by roughly a factor of 30 due to larger $Q$-factors, as compared to the optical band. Thus, the method proposed in this letter holds the promise of a higher accuracy than obtainable at present with QSO metal absorption lines.

Observations of both [C II] and CO lines in quasars and high-redshift galaxies will be one of the key tasks of the the Stratospheric Observatory for Infrared Astronomy (SOFIA) ${ }^{3}$, the Herschel Space Observatory (HSO $)^{4}$, and the Atacama Large Millimeter Array (ALMA) ${ }^{5}$. which are widely discussed in the literature. Thus, we can expect that these upcoming observations will contribute much to clarifying whether fundamental constants vary with cosmic time or not.

Acknowledgements. S.A.L., M.G.K., and S.G.P. gratefully acknowledge the hospitality of Hamburger Sternwarte while visiting there. This research has been partly supported by the DFG projects SFB 676 Teilprojekt C and RE 353/48-1, the RFBR grants No. 05-02-16914, 06-02-16489, and 07-02-00210, and by the Federal Agency for Science and Innovations grant NSh 9879.2006.2.

\section{References}

Abel, N. P. 2006, MNRAS, 368, 1949

Bahcall, J. N., Steinhardt, C. L., \& Schlegel, D. 2004, ApJ, 600, 520

Barvainis, R., Maloney, P., Antonucci, R., \& Alloin, D. 1997, ApJ, 484, 695

Bertoldi, F., Cox, P., Neri, R., et al. 2003, A\&A, 409, L47

Blain, A. W., Frayer, D. T., Bock, J. J., \& Scoville, N. Z. 2000, MNRAS, 313 , 559

Boselli, A., Gavazzi, G., Lequeux, J., \& Pierini, D. 2002, A\&A, 385, 454

Carilli, C. L., Menten, K. M., Stocke, J. T., et al. 2000, Phys. Rev. Lett., 85, 5511

Carilli, C. L., Neri, R., Wang, R., et al. 2007, ApJ, 666, L9

Caselli, P., \& Meyers, P. S. 1995, ApJ, 446, 665

Chand, H., Srianand, R., Petitjean, P., et al. 2006, A\&A, 451, 45

Dent, T., Stern, S., \& Wetterich, C. 2007, Phys. Rev. D, 76, 063513

Drinkwater, M. J., Webb, J. K., Barrow, J. D., \& Flambaum, V. V. 1998, MNRAS, 295,457

Dzuba, V. A., \& Flambaum, V. V. 2007

[arXiv:0711.4428] (physics.atom-ph)

Dzuba, V. A., Flambaum, V. V., Kozlov, M. G., \& Marchenko, M. 2002, Phys. Rev. A, 66, 022501

Falgarone, E., Puget, J. L., \& Perault, M. 1992, A\&A, 257, 715

Fan, X., Strauss, M., Schneider, D., et al. 2003, AJ, 125, 1649

Flambaum, V. V. 2007, Int. J. Mod. Phys., 22, 4937

Flambaum, V. V., \& Kozlov, M. G. 2007, Phys. Rev. Lett., 98, 240801

Frieswijk, W. W. F., Spaans, M., Shipman, R. F., Teyssier, D., \& Hily-Blant, P. 2007, A\&A, 475, 263

Fujii, Y. 2005, Phys. Lett. B, 616, 141

García-Berro, E., Isern, J., \& Kubyshin, Y. A. 2007, A\&A Rev., 14, 113

Gould, C. R., Sharapov, E. I., \& Lamoreaux, S. K. 2006, Phys. Rev. C, 74, 024607

Ho, P. T. P., Moran, J. M., \& Lo, K. Y. 2004, ApJ, 616, L1

Ichikawa, K., Kanzaki, T., \& Kawasaki, M. 2006, Phys. Rev. D, 74, 023515

\footnotetext{
3 http://www.sofia.usra.edu/

4 http://herschel.esac.esa.int/

5 http://www . eso.org/alma/
} 
Iono, D., Yun, M. S., Elvis, M., et al. 2006, ApJ, 645, L97

Kanekar, N., Carilli, C. L., Langston, G. I., et al. 2005, Phys. Rev. Lett., 95, 26130

Kaufman, M. J., Wolfire, M. G., Hollenbach, D. J., \& Luhman, M. C. 1999, ApJ, 527,795

Kaufman, M. J., Wolfire, M. G., \& Hollenbach, D. J. 2006, ApJ, 644, 283

Langacker, P., Segrè, G., \& Strassler, J. 2002, Phys. Lett. B, 528, 121

Larson, R. B. 1981, MNRAS, 194, 809

Levshakov, S. A. 1994, MNRAS, 269, 339

Levshakov, S. A., Molaro, P., Lopez, S., et al. 2007a, A\&A, 466, 1077

Levshakov, S. A., Agafonova, I. I., Molaro, P., \& Reimers, D. 2007b, A\&A, submitted

Liszt, H., \& Lucas, R. 2000, A\&A, 355, 333

Loeb, A. 1993, ApJ, 404, L37

Mac Low, M.-M., \& Klessen, R. S. 2004, Rev. Mod. Phys., 76, 125

Maiolino, R., Cox, P., Caselli, P., et al. 2005, A\&A, 440, L51

Maiolino, R., Neri, R., Beelen, A., et al. 2007, A\&A, 472, L33

Malhotra, S., Kaufman, M. J., Hollenbach, D., et al. 2001, ApJ, 561, 766

Marciano, W. J. 1984, Phys. Rev. Lett., 52, 489

Miesch, M. S., \& Bally, J. 1994, ApJ, 429, 645

Molaro, P., Levshakov, S. A., Monai, S., et al. 2007, A\&A [arXiv:0712.3345]

Mosquera, M. E., Scóccola, C. G., Landau, S. J., \& Vucetich, H. 2008, A\&A, 478,675

Mota, D. F., \& Barrow, J. D. 2004, MNRAS, 349, 291

Murphy, M. T., Webb, J. K., Flambaum, V. V., et al. 2001, MNRAS, 327, 1244
Nagamine, K., Wolfe, A. M., \& Hernquist, L. 2006, ApJ, 647, 60

Omont, A., Petitjean, P., Guilloteau, S., et al. 1999, Nature, 382, 428

Papadopoulos, P. P., Thi, W.-F., \& Viti, S. 2004, MNRAS, 351, 147

Peik, E., Lipphardt, B., Schnatz, H., et al. 2006 [arXiv:physics/0611088]

Petrosian, V., Bahcall, J. N., \& Salpeter, E. E. 1969, ApJ, 155, L57

Petrov, Yu. V., Nazarov, A. I., Onegin, M. S., Petrov, V. Yu., \& Sakhovsky, E. G. 2006, Phys. Rev. C, 74, 064610

Petuchowski, S. J., \& Bennett, C. L. 1993, ApJ, 405, 591

Pety, J., Beelen, A., Cox, P., et al. 2004, A\&A, 428, L21

Porsev, S. G., Koshelev, K. V., Tupitsyn, I. I., et al. 2007, Phys. Rev. A, 76, 052507

Redfiled, S., \& Linsky, J. L. 2008, ApJ, 673, 283

Schaeres, D., \& Pelló, R. 2005, in The dusty and molecular universe: a prelude to Herschel and ALMA, ed. A. Wilson (Noordwijk, The Netherlands: ESA Pub. Division), 121

Solomon, P. M., Rivolo, A. R., Barrett, J., \& Yahil, A. 1987, ApJ, 319, 730

Stacey, G. J., Viscuso, P. J., Fuller, C. E., \& Kurtz, N. T. 1985, ApJ, 289, 803

Stark, A. A. 1997, ApJ, 481, 587

Suginohara, M., Suginohara, T., \& Spergel, D. N. 1999, ApJ, 512, 547

Tzanavaris, P., Murphy, M. T., Webb, J. K., Flambaum, V. V., \& Curran, S. J. 2007, MNRAS, 374, 634

Varshalovich, D. A., \& Potekhin, A. Y. 1996, Astron. Lett., 22, 1

Wagg, J., Wilner, D. J., Neri, R., Downes, D., \& Wiklind, T. 2006, ApJ, 651, 46

Weiß, A., Henkel, C., Downes, D., \& Walter, F. 2003, A\&A, 409, L41

Weiß, A., Downes, D., Henkel, C., \& Walter, F. 2005, A\&A, 429, L25

Walter, F., Carilli, C., Bertoldi, F., et al. 2004, ApJ, 615, L17 